\title{
Use Artificial Neural Network Neococcontron in Distinguishing Handwritten Arabic Numerals
}

Laheeb Mohammad Ibrahim

Hanan H. Ali

College of Computer Sciences and Mathematics

University of Mosul, Iraq

\section{Received on: 21/09/2008}

\section{ABSTRACT}

Accepted on: 04/12/2008

Artificial Neural Networks have wide applications now a day, Among these are in the field of pattern recognition and image processing. This is due to the fact that it has a good performance and advanced mathematical computation power particularly its flexible adaptation to parallelism technique. That is why this research is conducted for the recognition of hand written Arabic numbers $(0-9)$. Recognition artificial neural network is simulated the human eye for tracking the property of entered image (Feature extractor).

The systems examined on samples of Arabic numbers its performance was found to be balanced in spite of the variations in position and direction of the recognized number. Keywords: Artificial Neural Networks, pattern recognition, Neococcontron Artificial Neural Networks.

$$
\begin{aligned}
& \text { استخدام الثبكة العصبية الاصطناعية نيوكوكنترون في تمييز الارقام العربية المكتوبة يدوياً }
\end{aligned}
$$

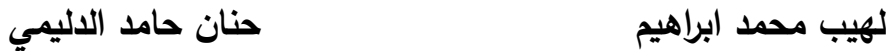

$$
\begin{aligned}
& \text { كلية علوم الحاسبات والرياضيات، جامعة الموصل }
\end{aligned}
$$

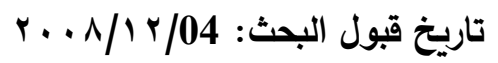

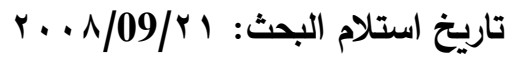

\section{الملخص}

ان الثبكات العصبية الاصطناعية ذات استخدامات واسعة جدا" في تمييز الأنماط ومعالجة الصور وذلك

لما تقدمه من كفاءة عالية في العمليات الحسابية وأسلوب عملها المتوازي لذلك تم في هذا البحث تصميم منظومة تمييز صورية للأرقام العربية (0_9) المكتوبة يدوياً وباستخدام النموذج العصبي الاصطناعي النيوكوكنترون ) Neocognitron )

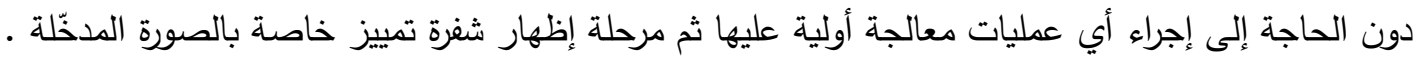
اختُر النظام على نماذج صورية للأرقام العربية وكان أداء النظام متوازناً بالرغم من التغير في موقع الرقم المراد تصنيفه ودورانه وكانت نسبة التمييز جيدة.

الكلمات المفتاحيه: الثبكات العصبيه الاصطناعيه، تمييز الانماط، الثبكه العصبيه الاصطناعيه نيوكونكترون 1 - المقدمة

لقد بدأ الاهتمام بحقل تمييز الأنماط منذ حقبة طويلة وأول حالة تمييز مسجلة عام (1929) تم الحصول عليها بواسطة العالم الألماني Tauschech ، اذ استخدم طريقة مطابقة القوالب(Template Matching) والتي اعتمد فيها على فكرة بسيطة من خلال مرور الضوء اذ يستخدم كاشف ضوئي للحصول على أقنعة (Masks ) معينة، وعندما يكون هناك تطابق كامل للحرف عندها لا يوجد ضوء يمر خلال القناع (mask) وبالتالي لا يصل ضوء للكاشف الضوئي، استمر العمل بهذه الفكرة لاكثر من (70) سنة، تبع هذه الطريقة فيما بعد طريقتين أخرتين

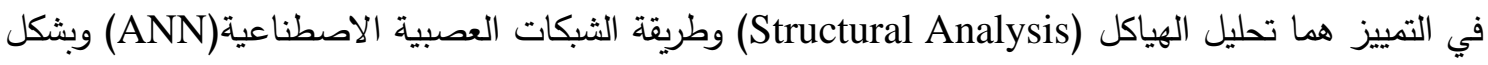
عام تم استخدام طريقة مطابقة القوالب في تمييز الصور المتمثلة في تمييز الحروف المطبوعة بينما تم استخدام 
طريقة تحليل الهياكل والثبكات العصبية الاصطناعية في تمييز الصور المتمثلة بالحروف المكتوبة يدويا". [2]

ان غالبية الأساليب المتبعة في عملية تمييز الصور تعمل على مبدأ اقتباس أولي لخواص الصورة

المدخلة ثم يتم بعدها تصنيف هذه الخواص لتوليد شفرة خاصة لصورة معينة، ان الغاية الأساس من مرحلة اقتباس

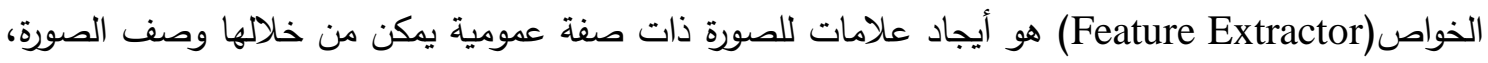
أي يمكن وصف الصورة بعدد محدد من العلامات وعلى أساس هذه الصفات العامة يمكن تصنيف عدد كبير من الصور .

بعض الباحثين استخدموا طريقة تكوين الفضاء الوهمي [3] لإيجاد الصفات الأساسية للصورة ثم تصنيف

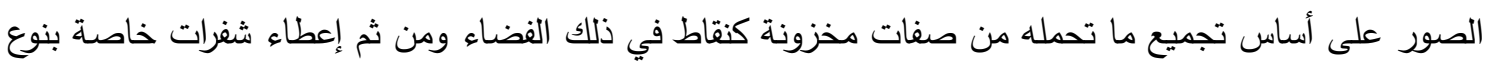
تلك الصور، اما مجموعة أخرى من الباحثين فقد أفادوا من الكفاءة التي أظهرتها الثبكات العصبية الاصنطناعناعية

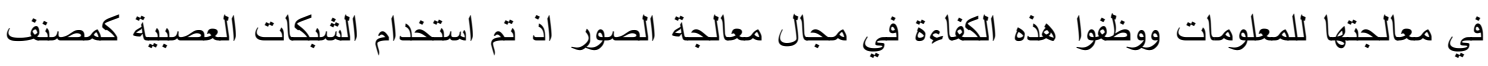
للمعلومات ومنها الصور وذلك بعد استخدام إحدى طرق اقتباس الخواص للحصول على صفات الصورة ومنها

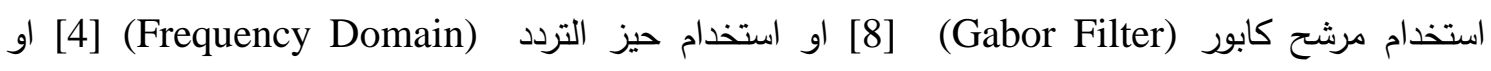

استخدام تحويل الموجة (Wavelet). هذه الطرق السابقة هي طرق هجينه تستخدم مع الثبكات العصبية الاصطناعية اذ يتم بهذه الطرق الحصول على خواص الصور ثم تدخل على الثبكات العصبية الاصطناعية لتصنيفها على أساس تكوين شفرة خاصة لكل صورة بعد تدريب الشبكة العصبية الاصطناعية. بعد ذلك تم تطوير بعض النماذج للشبكات العصبية الاصطناعية لتكون

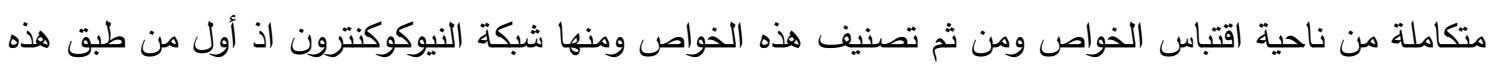

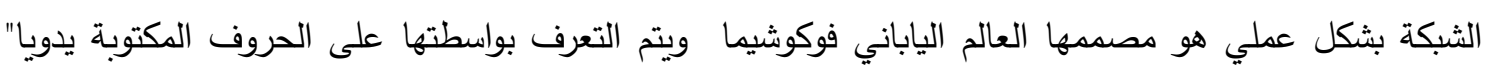
والأرقام العربية(0,1,2,3,4,5,6,7,8,9) وهدف الثبكة هو جعل استجابتها غير حساسة للاختلاف في موضع او

[7][2]. نمط الكتابة للأرقام

2-

ان عملية تمييز الصور تعد عملية غاية في الصعوبة اذ تكون هذه العملية متأثرة باحتمالية إزاحة الصورة

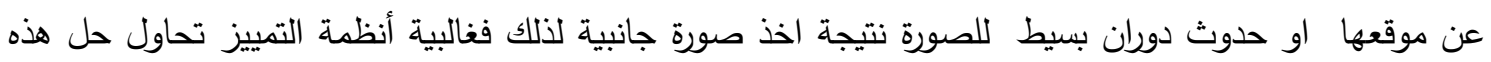
المشاكل بأن تكّون قاعدة بيانات كبيرة بحيث تشمل غالبية الاحتمالات التي قد تكون عليها وضعية الصورة المراد

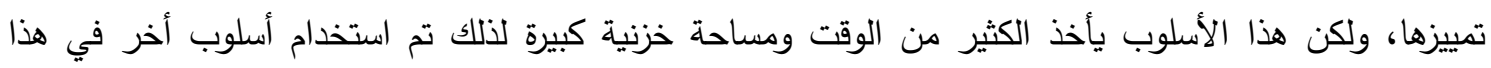

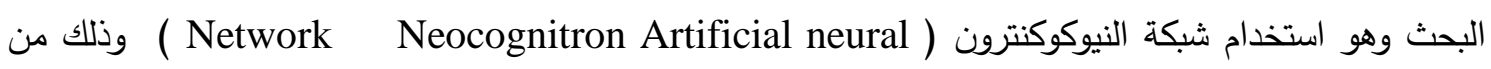
خلال محاكاة الخلية البايولوجية العاملة في عين الإنسان. ان استخدام شبكة النيوكوكنترون(Neocognitron)

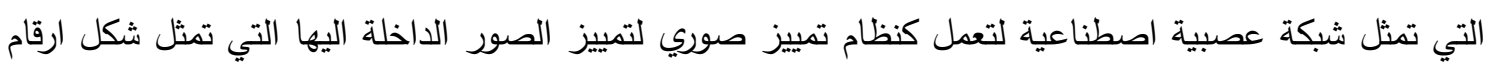

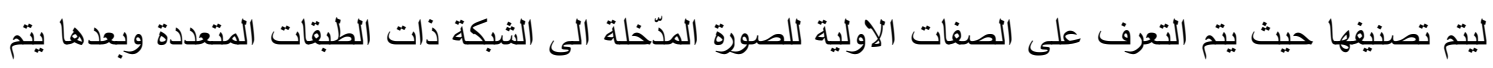
إعطاء شفرة خاصة للصورة المدّخلة وبذلك يتم تصنيفها، اذ ان النيوكوكنترون تمتلك من الصفات ما يمّكن الباحثين

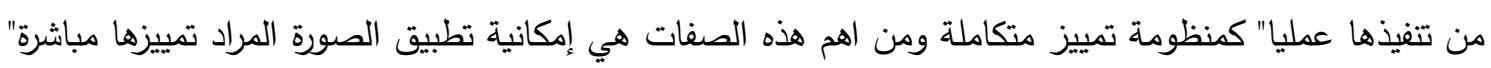
لذلك فالهدف من هذا البحث هو تصميم نظام تمييز صوري بالإفادة من مميزات علم الثبكات العصبية الاصطناعية واستخدمت شبكة النيوكوكنترون لأنها قادرة على التمييز الصوري بين صور تتنمي لأصناف مختلفة، 
وقدرة هذه الثبكة على تصنيف اكبر عدد ممكن من الصور إلى الصنف الذي تتنمي إليه بشكل صحيح حتى إذا

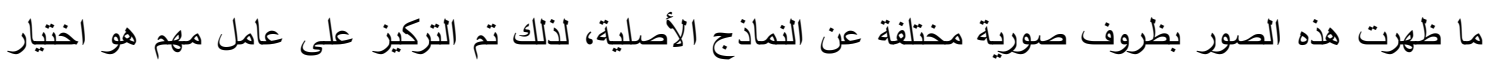
شبكة عصبية اصطناعية مناسبة التي تكون قادرة على التدرب على الصور الأصلية فقط دون الحاجة الى استخدام صور ذات إزاحة صغيرة عن الصورة الأصلية او تغيير في حجم الصورة اذ تكون هذه الثبكة قادرة على التغلب التبل على اغلب المشاكل آنفة الذكر اذ تمتلك هذه الثبكة صفة التغذية الأمامية (feed forward) وهي ذات ذات طبقات متعددة يجعلها تمتلك إمكانية عالية في التصنيف لذلك تستخدم بشكل واسع في تمييز الصور والكتابة اليدوية، أي

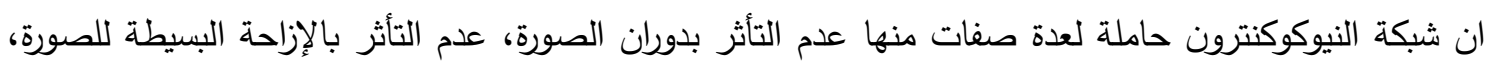
عدم التأثر بالتغيرات على الإضاءة، عدم التأثر الكبير بالتشويه في الصورة الحاصل نتيجة حركة الجسم أثناء اخذ

[13][11] [1]

\section{3- 3 الشبكة العصبية الاصطناعية النيوكوكنترون}

تعرف الثبكات العصبية على انها العلم الذي يهتم بدراسة الأساليب الرياضية التي يمكن صياغتها

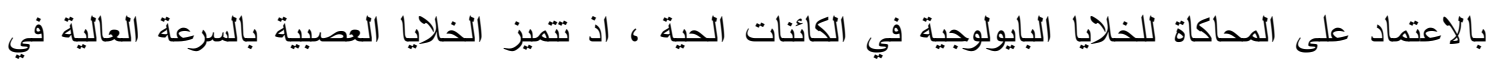

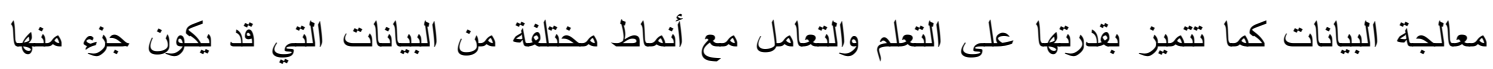

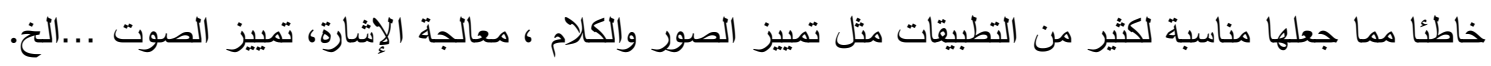
الشبكات العصبية الاصطناعية عبارة عن هيكل ذو بناء متوازي المعلومات، يتكون هذا الهيكل من وحدات معالجة هئل

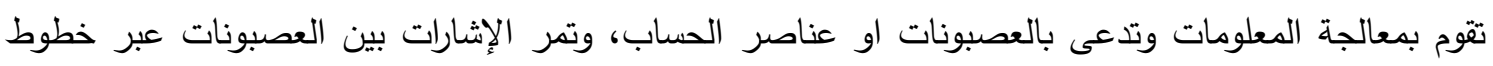

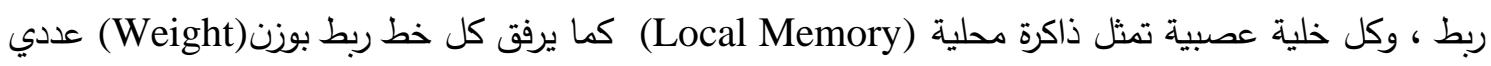
معين يضرب مع الاثارات الداخلة للعصبون ثم يطبق على كل عصبون دالة تفعيل ( Activation Function)

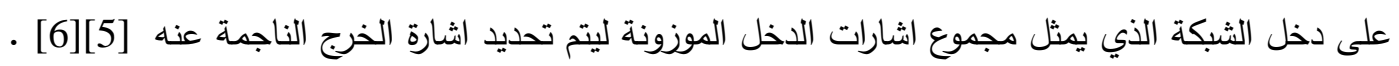
تعد شبكة النيوكوكنترون تطويرلشبكة بدائية اطلق عليها اسم ”Cognitron"، وهي نوع من شبكات التتظيم الذاتي، اذ أول من استخدم هذا النوع من الشبكات وكما تقدم هو العالم الياباني فوكوشيما عام (1975) وتعد هذه الثبكات مثالا" للشبكة الهرمية التي تتكون من طبقات عدة وباتصالات محلية متفرقة ما بين الطبقات، وفي عام (1983) طورت شبكة النيوكوكنترون ذات التدريب الموجه عن الشبكة الاصلية الـ"Cognitron" ، [2][2][2] اذ تتألف البنية الهندسية لثبكة النيوكوكنترون من اربع طبقات زوجية اضافة" الى طبقة الدخل

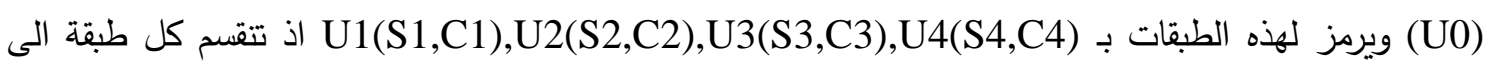
طبقتين فرعيتين الطبقة الفرعية البسيطة S Cتبعها الطبقة الفرعية المركبة C وتترتب الوحدات في كل طبقة وفق مصفوفات مربعة اذ يتم تدريب المصفوفات في (S) للاستجابة لعينة خاصة او مجموعة من العينات ـ اما الطبقة فتقوم بتجميع النتائج الخاصة بها من العلاقة التي تربطها مع مصفوفات الطبقة (S) (C) يتم التقليل من عدد الوحدات في كل مصفوفة الى ان نصل الى طبقة الاخراج (Output Layer) اذ يؤخذ الاخراج من الطبقة C4 ،لاحظ الثكل (1). ان شبكة النيوكوكنترون تعد من الثبكات المتعددة الطبقات وذات تغذية امامية

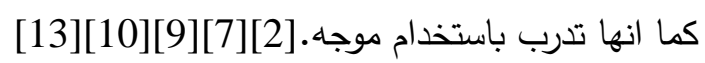


ان تصميم شبكة النيوكوكنترون مكون من طبقة الدخل (Input Layer) اذ يتم ترتيب طبقة الدخل وفق

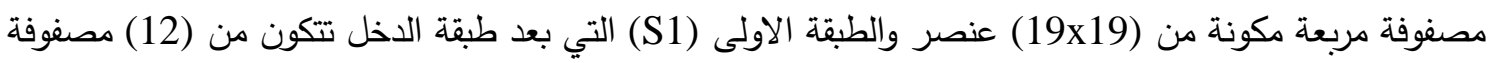

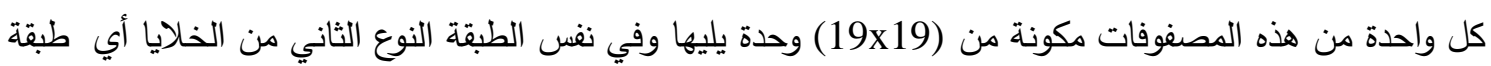

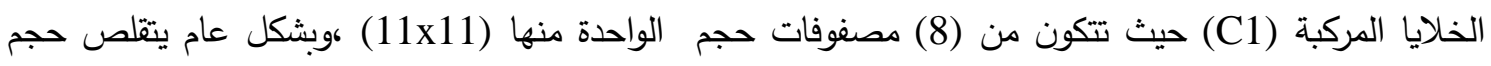

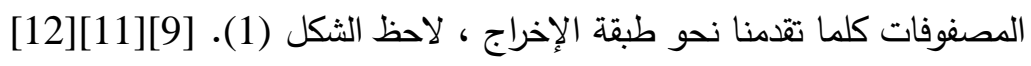

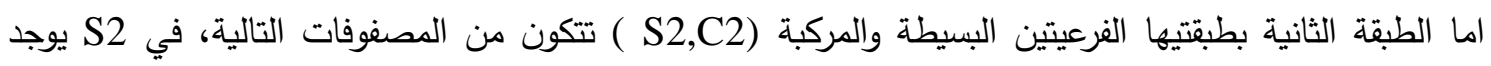
(38) مصفوفة ذات حجم (11x11) اما C2 فتتكون من (22) مصفوفة ذات حجم (11x11) ، اما الطبقة الثالثة فتتكون الطبقة الفرعية البسيطة فيها من (32) مصفوفة بحجم (7x3,C3)

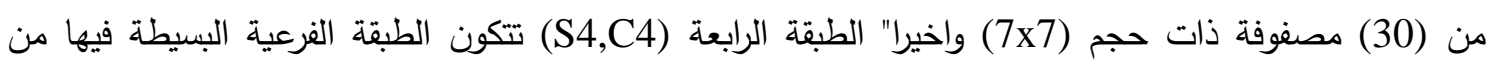

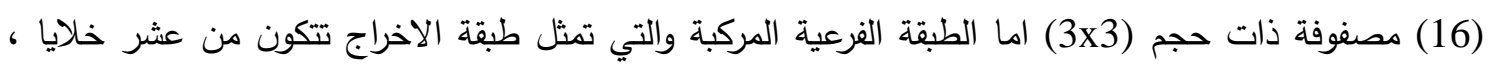

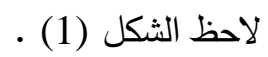
في الحقيقة تستقبل كل وحدة في المصفوفات التابعة للطبقة الفرعية المركبة ( C1 ) من الطبقة الاولى اشاراتها من

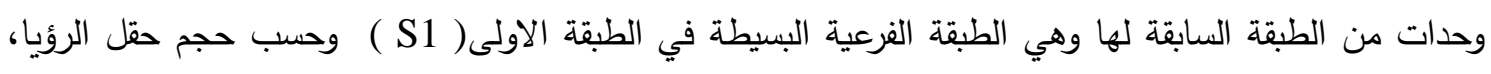

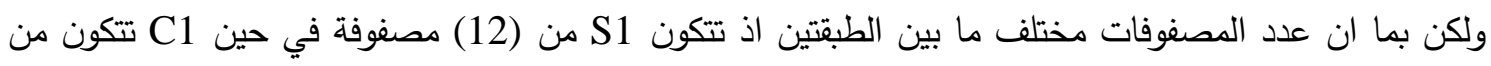

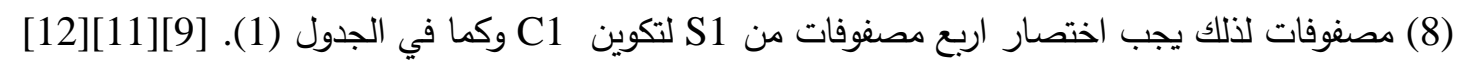

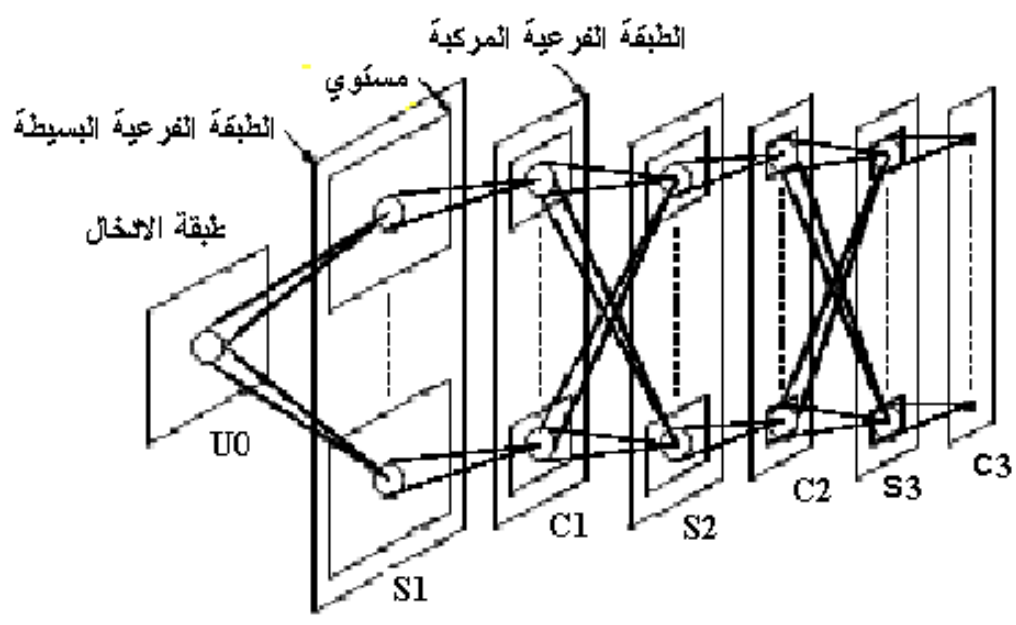

الشكل (1) تصميم شبكة نيوكوكنترون

الجدول (1) الترابطات القادمة من S1 الى C1)

\begin{tabular}{|lll|}
\hline $\operatorname{S1}(1)$ & $\rightarrow$ & $\mathrm{C1}(1)$ \\
$\operatorname{S1}(2), \mathrm{S1}(3)$ & $\rightarrow$ & $\mathrm{C1}(2)$ \\
$\operatorname{S1} 1(4)$ & $\rightarrow$ & $\mathrm{C} 1(3)$ \\
$\operatorname{S1}(5), \mathrm{S1}(6)$ & $\rightarrow$ & $\mathrm{C1}(4)$
\end{tabular}




\begin{tabular}{|lll|}
\hline $\mathrm{S} 1(7)$ & $\rightarrow$ & $\mathrm{C} 1(5)$ \\
$\mathrm{S} 1(8), \mathrm{s} 1(9)$ & $\rightarrow$ & $\mathrm{C} 1(6)$ \\
$\mathrm{S} 1(10)$ & $\rightarrow$ & $\mathrm{C} 1(7)$ \\
$\mathrm{S} 1(11), \mathrm{s} 1(12)$ & $\rightarrow$ & $\mathrm{C} 1(8)$ \\
\hline
\end{tabular}

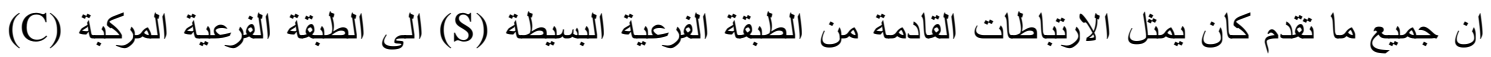

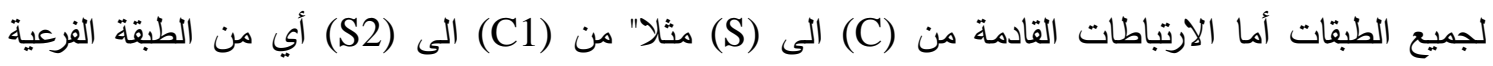

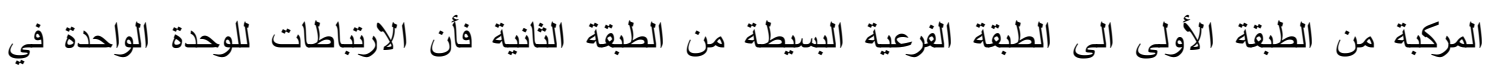

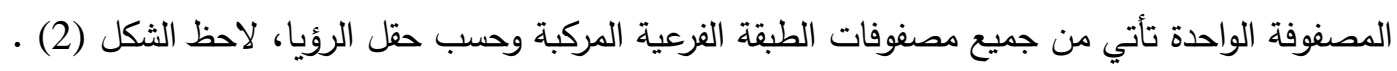

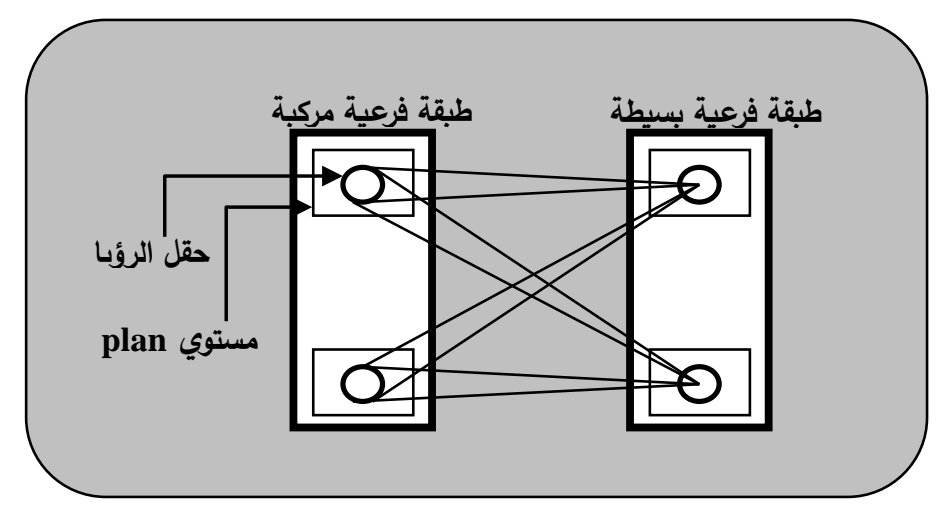

الثكل (2) ارتباطات طبقة فرعية مركبة الى طبقة فرعية بسيطة

3-2 20 - 2قل الروئا

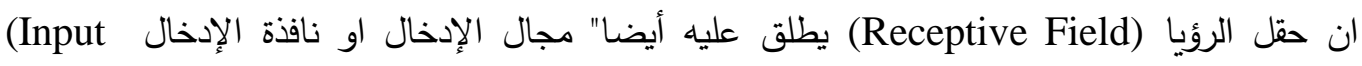

(1) مindow)

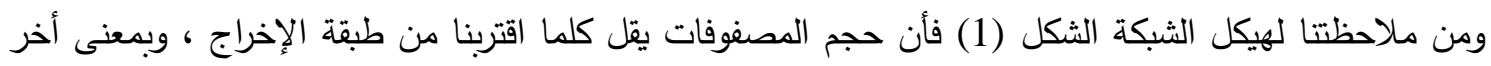

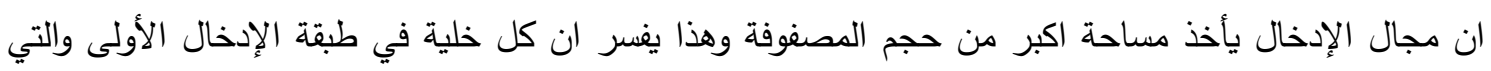

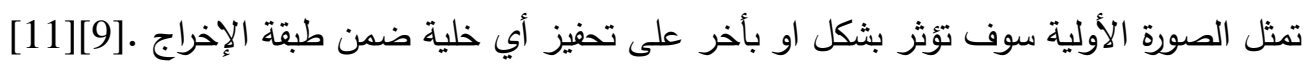
3-3 آلية عمل النيوكوكنترون في تمييز صور الأرقام العربية

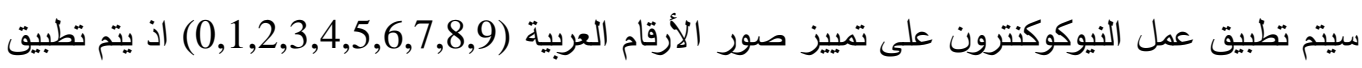

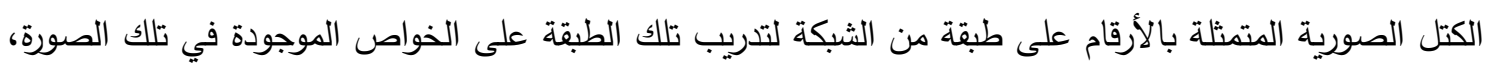

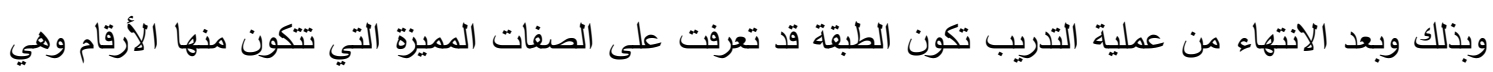

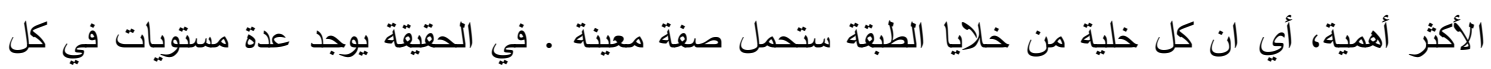

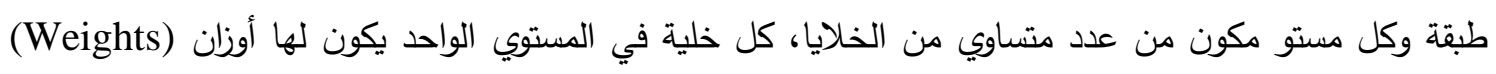

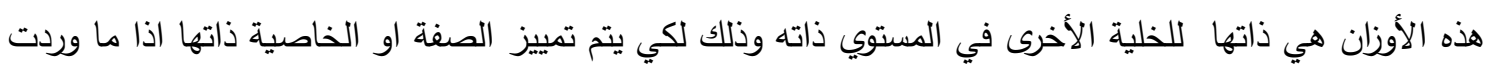

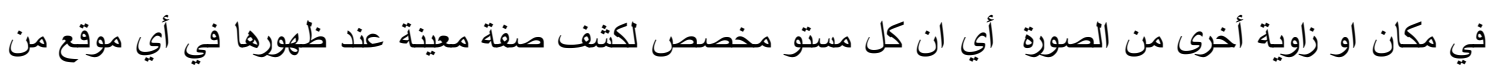

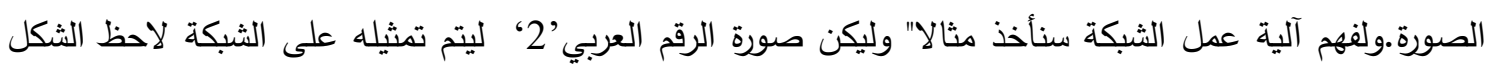


سيتم إدخال الرقم من طبقة الإدخال (Input Layer) الى الطبقة الأولى المتمثلة بالطبقة الفرعية البسيطة والمركبة (S1,C1) اذ يتم هنا اقتباس الصفات الأولية للصورة وبما انه يوجد عدة مستويات فأن كل مستوي سيكثف صفة معينة فمثلا" المستوي الأول للطبقة الفرعية البسيطة S1 سيتم اقتباس الجزء العلوي من الرقم وهو ( ) وذلكهـ لان الخلية التي في الجزء العلوي ستقتبس هذه الصفة وكما في الثكل(4).

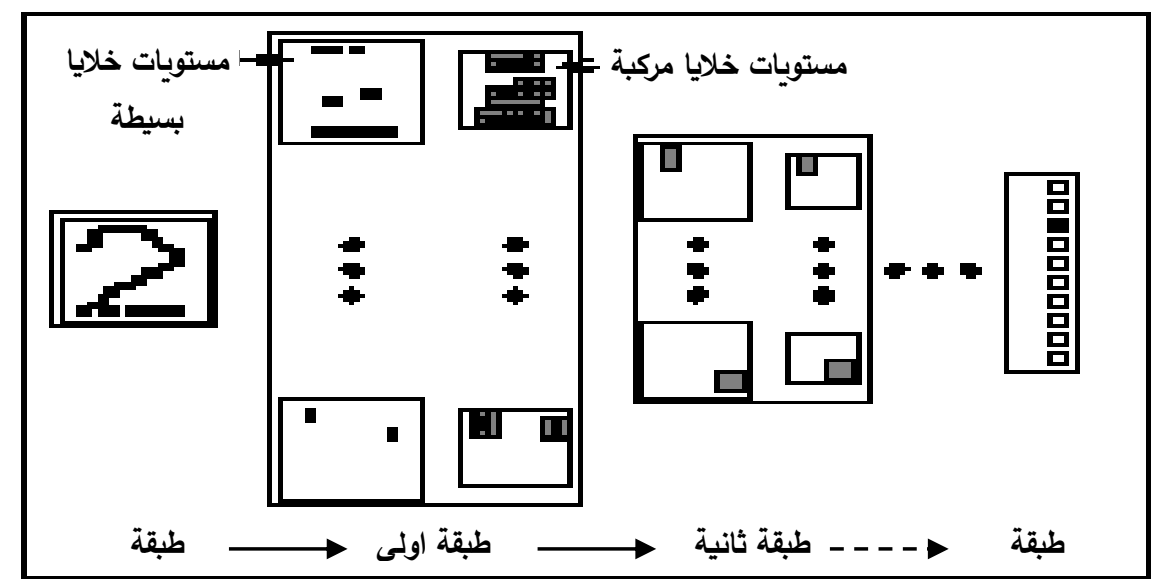

الثكل (3) الهيكل العام لثبكة النيوكوكنترون لكثف الرقم " 2 "

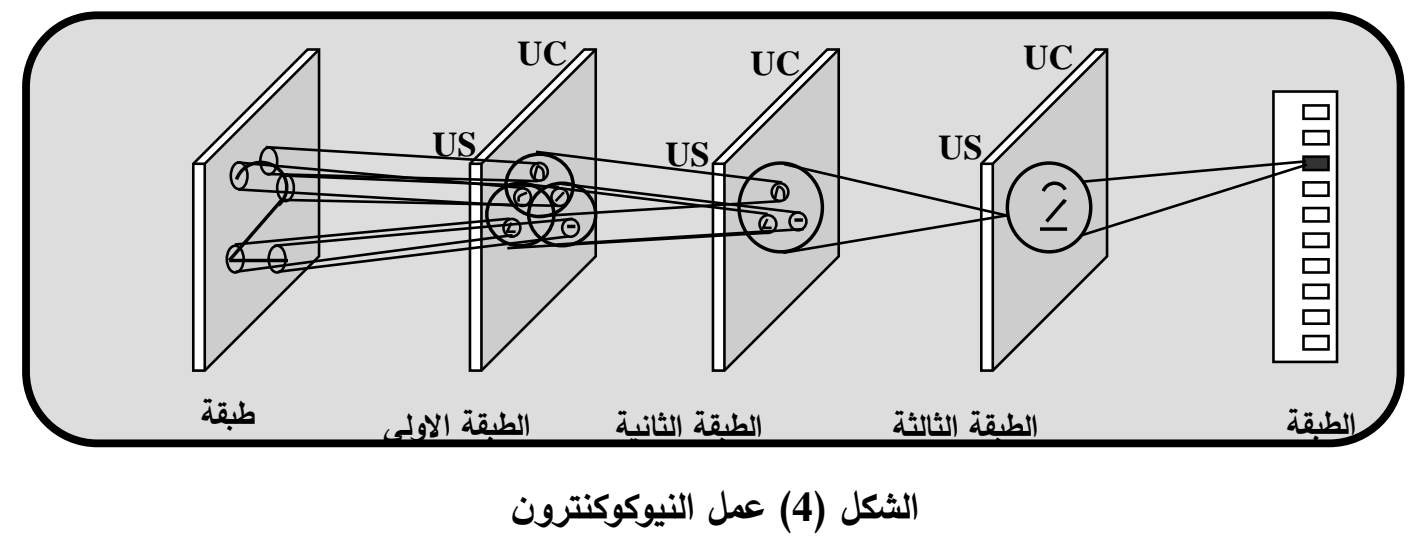

ثم المستوي الثاني يأخذ صفة اخرى (I ) والمستوي الثالث يأ خذ الصفة ج) ) وهكذا مع ملاحظة انه اذا ما كانت

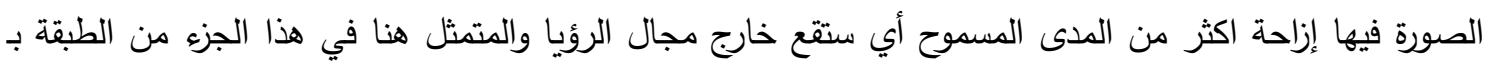
(3x3) ، عند ذلك سيتم إعطاء صفات مختلفة عن الصورة الأصلية كما في بعض مستويات الثكل (5) (5).

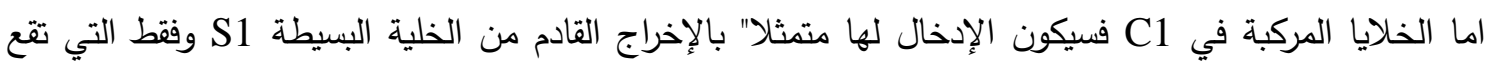

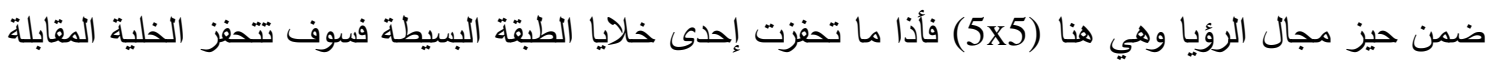
في الطبقة المركبة أي ستستجيب لذات الصفة اذا ما ظهرت في مجال الرؤيا لها أي مجال الإدخال، ثم تأتي الطبقة الثانية متمثلة بالخلايا البسيطة والمركبة (S2,C2) وعلى ذات المبدأ ستعمل كما عملت الطبقة السابقة لها

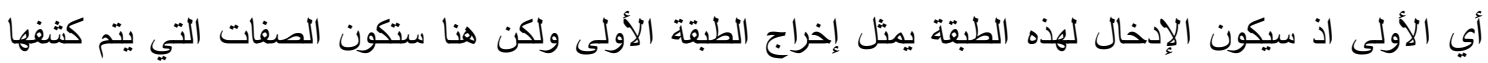

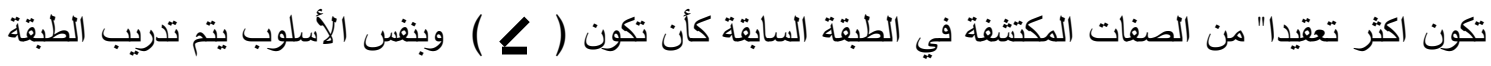
الثالثة ايضا" والتي تكون قادرة على كثف الصفات المعدة مثلا"ه ) وهكذا الى ان يتم تمييز الرقم ( 2 ) بأكمله 
في ما لو ظهر في أي موقع ضمن الإدخال وبذلك تخرج النتيجة ضمن الطبقة الرابعة والتي يكون كل مستوي فيها

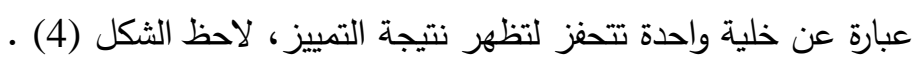

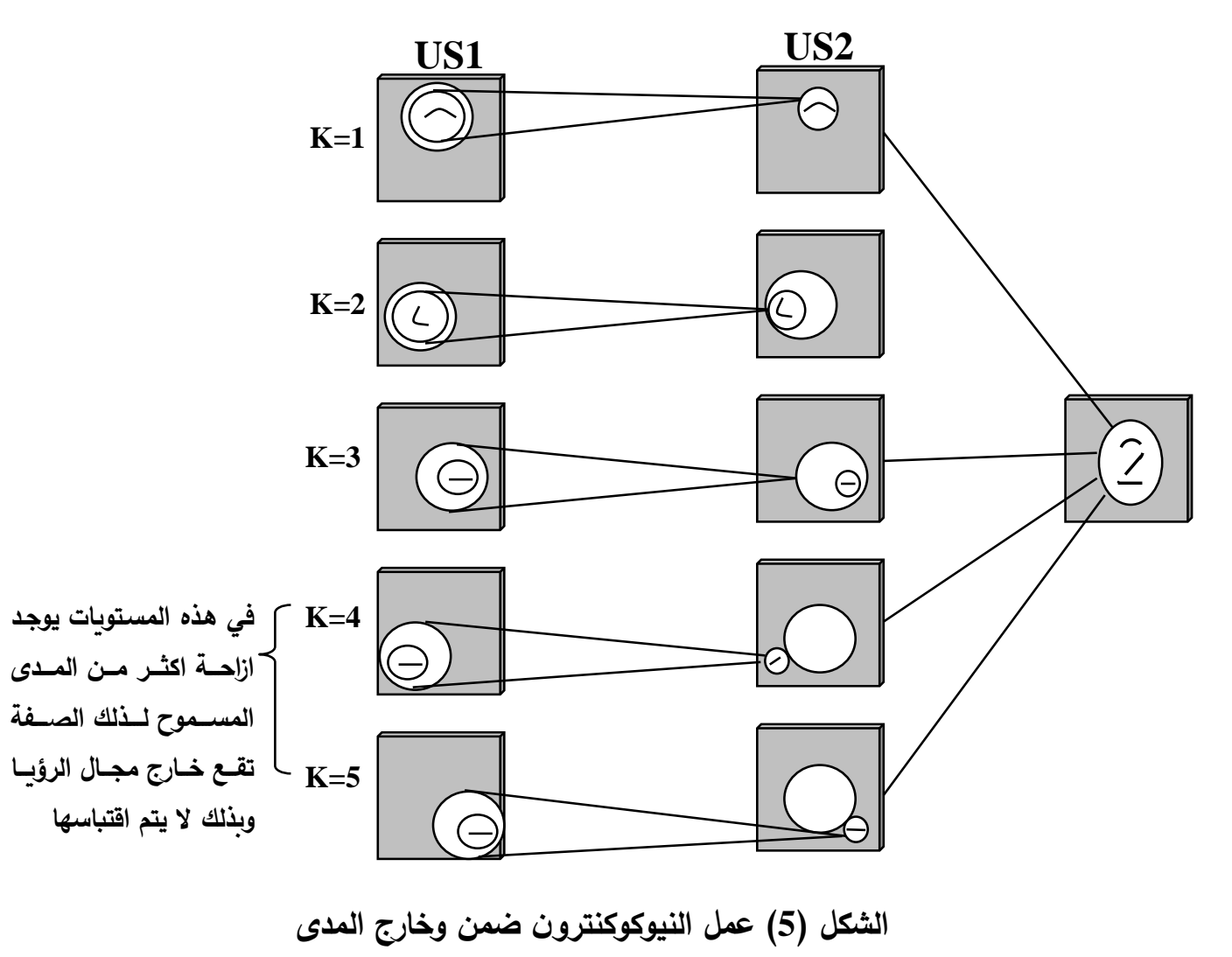

4- هيكلية بناء نظام التمييز باستخدام الثبكة العصبية الاصطناعية

عند بناء نظام يقوم بعملية تمييز صوري باستخدام الثبكات العصبية الاصنطناعية عليه ان يأخذ بنظر الاعتبار

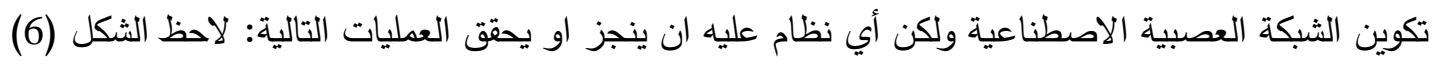
1- يجب ان يحتوي على عدد من النماذج (samples) للصور التي يمكن ان تحمل او تقارن معها من معمارية قاعدة بيانات (data base) منجزة سابقاً إضافة" إلى الأوزان التابعة لهذه النماذج.

2- المعلومات المطلوبة عن معمارية الشبكة العصبية في قاعدة البيانات يجب ان تتضمن معلومات عن حقل الرؤيا للطبقة كما يتضمن خزن قيم الأوزان الخاصة بالوحدات خصوصاً الطبقات s لأنها هي التي تتدرب فئس وبالتالي تتعلم. 


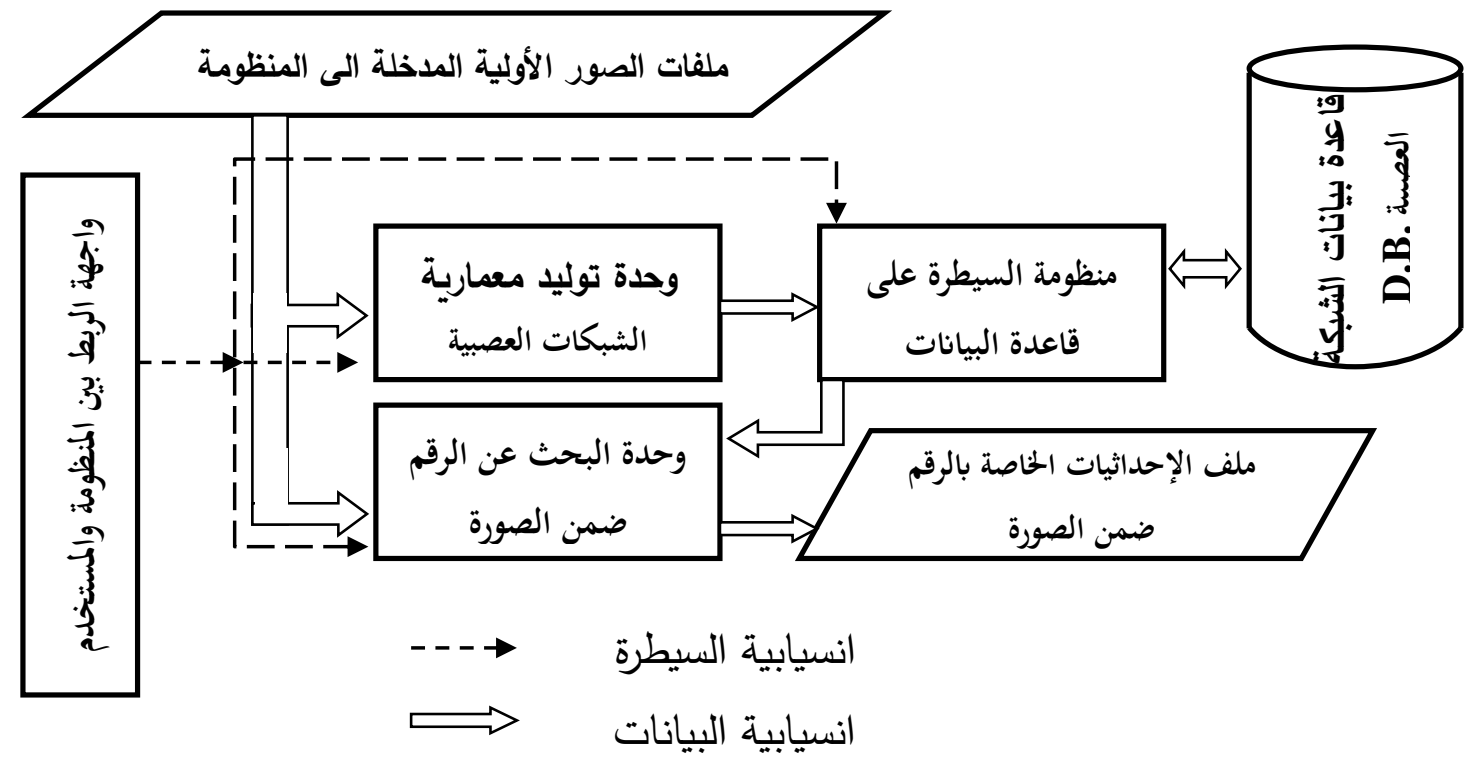

الشكل (6) هيكلية نظام برمجي للتمييز

$$
\text { 5- التطبيق العملي والنتائج }
$$

ان عملية تعليم شبكة النيوكوكنترون تمر بالخطوات التالية:

الخطوة الأولى: اختيار أرقام عشوائية صغيرة لاوزان الارتباط Wi,j بواسطة دالة تبلة تولد الأرقام العشوائية.

Target (input vector) الخطوة الثانية: تحديد زوج التدريب من متجه الإدخال الإخراج المتوقع الإنه

.(vector

الخطوة الثالثة: يتم حساب إخراج الوحدات في الطبقة الفرعية البسيطة Simple cells) S من الطبقة الواحدة وذلك باستخدام المعادلة التالية:

$$
\begin{aligned}
& x=\frac{1+e}{1+h}-1 \\
& e=\sum_{i} c_{i} \cdot w_{i} \\
& h=v \cdot w_{o}
\end{aligned}
$$

e: تمثل مجموع الإدخالات بعد ضربها بأوزانها، h الإدخال الكبحي الوحيد الموجب للخلية[1]

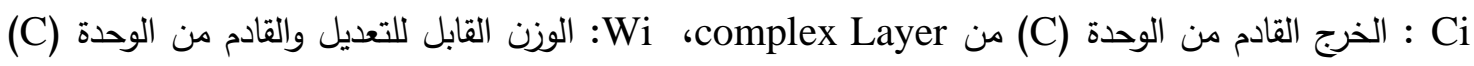

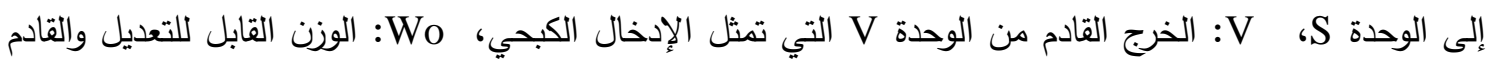

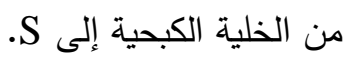
ثم يتم إدخال هذا الإخراج (X) على دالة التفعيل لتعطي إشارة الخرج للخلية (S) وفق العلاقة: $F(x)=\left\{\begin{array}{cc}x & \text { if } x \geq 0 \\ 0 & \text { if } x<0\end{array}\right.$ 
مع ملاحظة ان (Ci) في معادلة حساب قيمة e بالنسبة للطبقة الفرعية البسيطة (S1) للطبقة الأولى يمثل طبقة

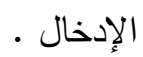
الخطوة الرابعة: يتم حساب إخراج الوحدات في الطبقة الفرعية المركبة Complex cells) C للطبقة الواحدة

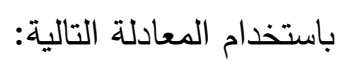

$$
\begin{aligned}
& x=\frac{1+e}{1+h}-1 \\
& e=\sum_{i} S_{i} \cdot W_{i}
\end{aligned}
$$

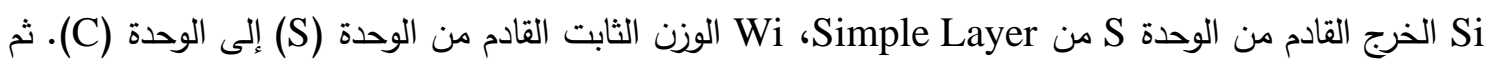
يتم إدخال هذا الإخراج (X) على دالة التفعيل والتي هنا تكون مختلفة عن دالة التفعيل في الطبقة الفرعية البسيطة S لتعطي إثارة الخرج وفق العلاقة

$\mathrm{F}(\mathrm{x})= \begin{cases}\mathrm{o} \geq \mathrm{if} \mathrm{x} & \frac{e}{a+e} \\ & \text { if } \mathrm{x}<\mathrm{o}\end{cases}$

وتكون قيمة المعامل (a) على الطبقة اذ تكون قيمتها (0.25) بالنسبة للطبقة (3.2.1) وبقيمة (1) للطبقة (4)

الخطوة الخامسة: يتم مقارنة الإخراج الحقيقي مع الإخراج المطلوب واذا يوجد فرق بين الاخراجين يتم تغيير أوزان الثبكة باستخدام المعادلة التالية:

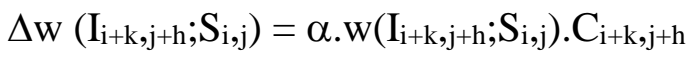

اذ ان C تمثل الطبقة السابقة وتكون W قيمة الوزن السابق وقيمة a تمثل قيمة نسبة التعلم وقيمتها اكبر من

الصفر

الخطوة السادة: يتم الرجوع إلى الخطوة الثالثة عند حدوث تغير بالاوزان .

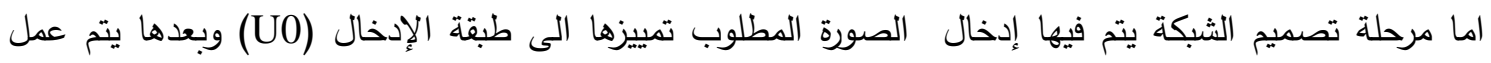

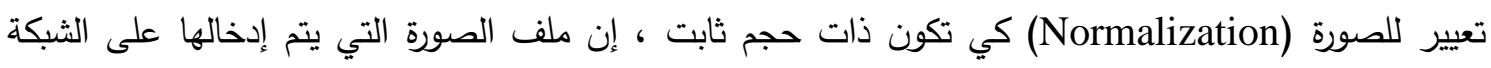
يكون من نوع (Bitmap (BMP) لأن هذا النوع من ملفات الصور ذات استخدام واسع أي يمثل صيغة قياسية

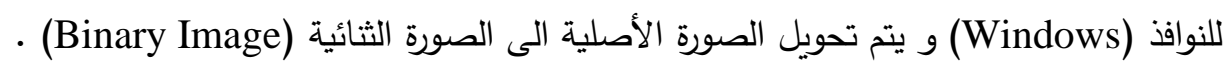
لقد تم جمع عينات قاعدة البيانات والتي تمثل الصور التي تتدرب عليها الشبكة من خلال تكوين صور تحمل شكل

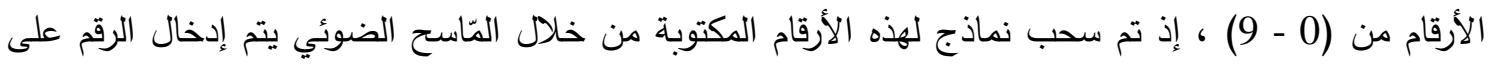
الثبكة وبذلك يكون هذا الرقم على شكل صورة من نوع (BMP) ، إذ يتم قراءة هذه الصورة داخل نظام التمييز

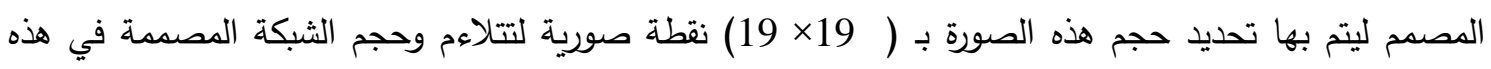
المنظومة .لاحظ المخطط الانسيابي الموضح في الثكل (7) يبين مراحل العمل: 


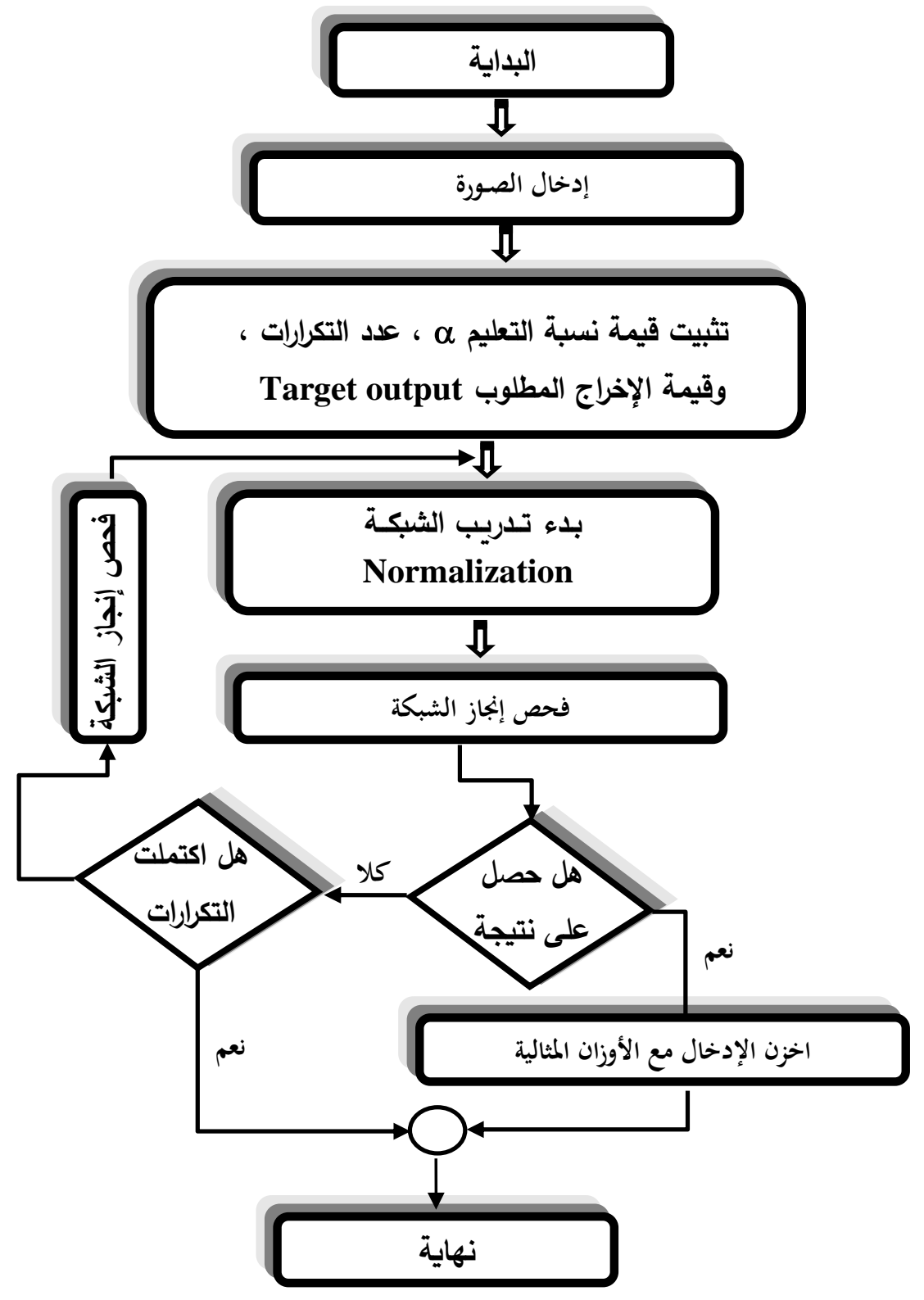

الثكل (7) المخطط الانسيابي لتصميم شبكة النيوكوكنترون

6- نسبة تمييز شبكة النيوكوكنترون

ان نسبة تمييز الثبكة تُحسب من خلال عدد الصور غير المصنفة بثنكل صحيح الى الصور الكلية وكما

في المعادلة التالية:

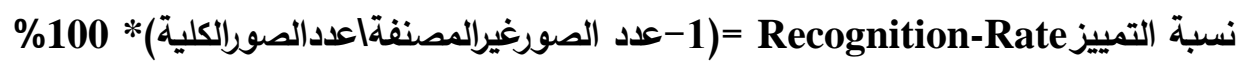

وقد تم اختبار عمل النظام على صور ارقام عربية مكتوبة باليد (100 صورة تمثل ارقام عربية بواقع 20 وثيقة قام

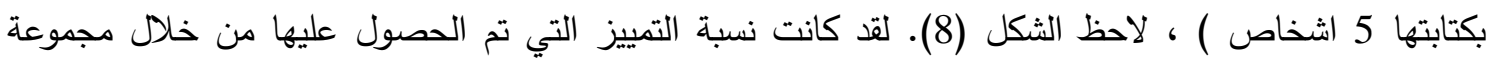

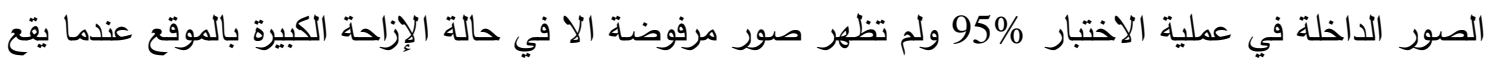

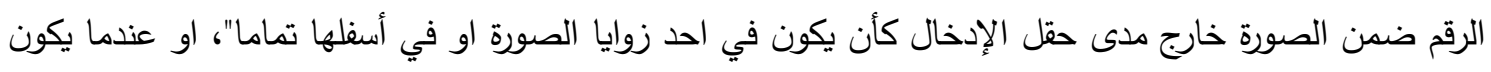

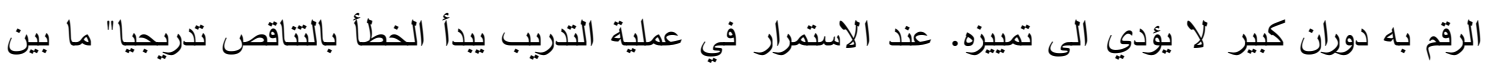


الإخراج الفعلي للشبكة والهدف المنشود وبذلك تكون الثبكة قد تمكنت من الحصول على قدرة تمييزية تمكنها من الفصل بين الأصناف المختلفة.

$\begin{array}{llllllllll}0 & 1 & 2 & 3 & 4 & 5 & 6 & 7 & 8 & 9 \\ 0 & 1 & 2 & 3 & 4 & 5 & 6 & 7 & 8 & 9\end{array}$

الشكل (8) يمثل بعض نماذج الأرقام المكتوبة بخط اليد

7- (الاستنتاجات

ان الغاية الاساسية من هذا البحث هو تصميم نظام تمييز صوري بالإفادة من مميزات علم الثبكات

العصبية الاصطناعية ( شبكة النيوكوكنترون) في التمييز ومن خلال التجارب التي قمنا بها وجدنا ان شبكة نيوكوكنترون قادرة على التمييز الصوري بين صور تتنمي لأصناف مختلفة، وقدرة هذه الثبكة على تصنيف اكبر عدد ممكن من الصور إلى الصنف الذي تتتمي إليه بشكل صحيح حتى إذا ما ظهرت هذه الصور بظروف صورئ صورية مختلفة عن النماذج الأصلية،كما انها قادرة على التدرب على الصور الأصلية فقط دون الحاجة الى استخدام صور

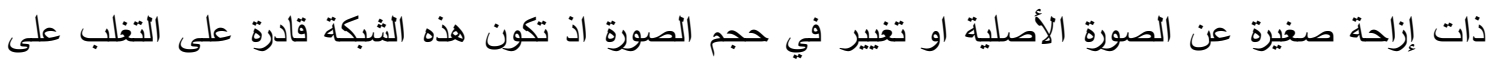
اغلب المشاكل آنفة الذكر وهي ذات طبقات متعددة يجعلها تمتلك إمكانية عالية في تمييز الصور والكتابة اليدوية، أي ان شبكة النيوكوكنترون حاملة لعدة صفات منها عدم التأثر بدوران الصورة، عدم التأثر بالإزاحة البسيطة

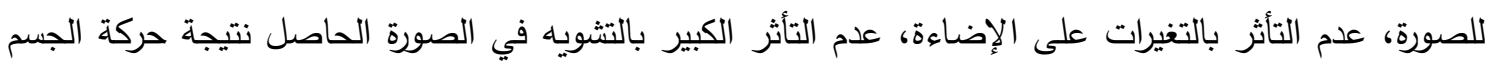
أثناء اخذ الصورةكما وجد ان عدد الخطوات التي تحتاجها الثبكة تزداد عندما تكون قيمة نسبة التعلم قليلة وتكون

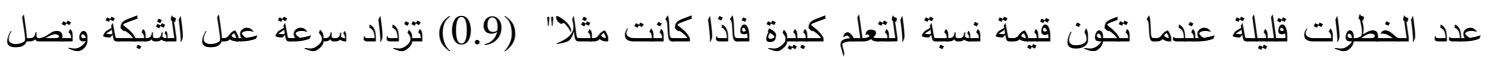
الى الحل بعدد أقل من الخطوات في حين تحتاج الى خطوات اكثر عندما تكون القيمة مثلا" (0.1) , لاحظ الجدول (2) ،والثكل (9) يوضح العلاقة ما بين قيمة نسبة التعلم مع عدد خطوات شبكة النيوكوكنترون في تمييز الارقام. 
الجدول (2) تأثير قيمة نسبة التعلم على عدد خطوات الثبكة

\begin{tabular}{|c|c|c|c|}
\hline عدد خلايا الادخال & عدد خلايا الاخراج & قيمة نسبة التعلم & عدد الخطوات \\
\hline 361 & 10 & 0.1 & 3311 \\
\hline 361 & 10 & 0.2 & 3280 \\
\hline 361 & 10 & 0.3 & 3207 \\
\hline 361 & 10 & 0.4 & 3081 \\
\hline 361 & 10 & 0.5 & 2771 \\
\hline 361 & 10 & 0.6 & 2562 \\
\hline 361 & 10 & 0.7 & 2351 \\
\hline 361 & 10 & 0.8 & 2100 \\
\hline 361 & 10 & 0.9 & 1820 \\
\hline
\end{tabular}

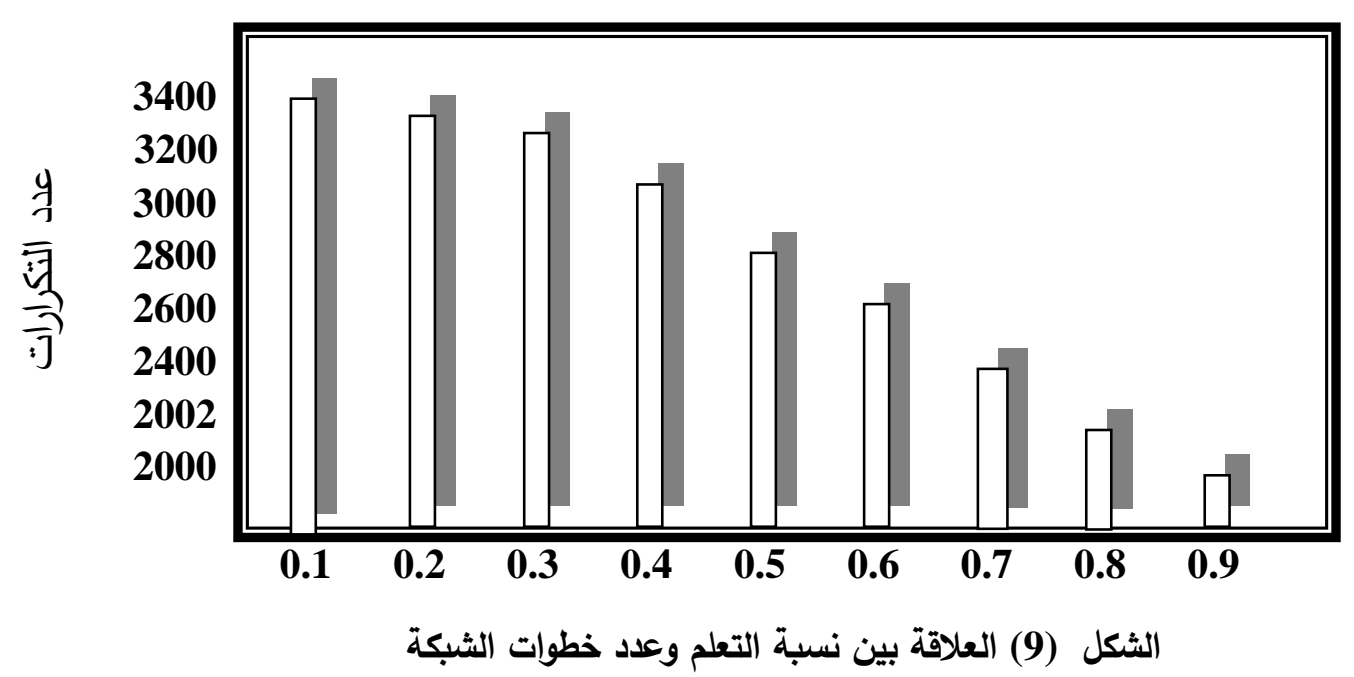

كما ان اختيار عدد دورات التدريب (Iteration) والذي يمثل زمن التدريب لتدريب الثبكة يجب ان يكون ملائماً لتطبيق كل النماذج المراد تدريبها فالزيادة الكبيرة بعدد دورات التدريب لن تكون ذا فائدة كبيرة وانما استهلاك للجهد والوقت كما ان اختيار عدد دورات التدريب قليل قد يؤدي الى عدم تدريب الثبكة ومن ثم عدم قدرتها على التمييز . ومن ملاحظة الجدول (3) يتبين مدى تأثر نسبة التمييز بعدد دورات التدربب عند التدربب فعندما تكون نسبة التمييز كبيرة نلاحظ ان عدد دورات التدريب كان اكبر من سابقه.

الجدول (3) علاقة نسبة التمييز بعدد التكرارات

\begin{tabular}{|r|c|c|c|c|}
\hline عدد دورات التدريب & 2000 & 2500 & 3000 & 3311 \\
\hline ع 185 & \%87.5 & \%90 & \%95 \\
\hline
\end{tabular}




$$
\begin{aligned}
& \text { المصادر } \\
& \text { الاليمي، حنان حامد علي ، (2003) ،" استخدام الثبكة العصبية الاصطناعية نيوكوكنترون في تمييز } \\
& \text { الأرقام العربية المكتوبة يدويا " ، أطروحة ماجستير ، جامعي ، جامعة الموصل. } \\
& \text { عيسى ، علام زكي ، (2002) ،" الثبكات العصبية - البنية الهندسية - الخوارزميات - التطبيقات " ، } \\
& \text { شعاع للنشر والعلوم ، سوريا. } \\
& \text { ياسين ، شفاء عبدالرحمن داؤد ، (2002) ،" تمييز الوجوه باستخدام الثبكة العصبية النيوكنترونية"، }
\end{aligned}
$$

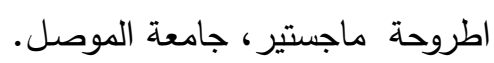

[4] Gonzalez, Rafael C , (1977), “ Digital Image Processing ”, Addison - Wesley Publishing Company .

[5] Kinnebrock, Werner, (1995), “Neural Networks Fumdoman tals , Application, example ‘, Galgotia publications, prt. 1td. , New Delhi .

[6] Luger G. F. and stubble field W. A. (1998), “ Artificial Intelligence structures and strategies for complex problem solving " Addison Wesley longman , Inc. , USA .

[7] Wasserman , P. D. , (1989), “ Neural computing theory and practice " , Van Nostramd Reinhold, New York .

[8] Chan , Khue-Hiang, “ Feature Extraction for Hand with Character Recognition", www.icsat.com/papers/ 102 mfi.pdf. Singapore .

[9] Lovell , D.R. , \& Tsoi Ah C. , (1992), “ The performance of the Neocognitron with Various S-cell and C-cell Transfer Functions “, citeseer.nj.com/ Lovell 92 performance . html.

[10] Lovell , D.R. , Downs , Tom , \& Tsoi , chc . , “An Evaluation of the Neocognitron“, www.eng.cam.ac.uk/ drl/publications/ieeTnn 95.abstract.html.

[11] Ooyen , A.van \& Nienhuis , B. , (1993), " pattern Recognition in the Neocognitron is Improved by Neuronal Adaptation“, http://www.GNC.ed.ac.uk/ people / arjen . html .

[12] SADYKHOV, R.Kh. \& VATKIN , M. E. , (2001), “ Algorithm for Image processing of integrated circuits basis of the Neocognitron Neural Network " , www.iie.uz.zgora.pl/archire/desdes01/files/ref/r-3.pdf.

[13] SATOH, S. , Kuroiwa J. , ASO,H.and Miyake,S.,'Recognitier of SATOH, S. , Kuroiwa J. , ASO,H.and Miyake,S.,'Recognitier of rotated patterns using neocognitron ", www.aso.ecei.tohoku.ac.jp/ shun/pdf/icoip97.pdf. 